\title{
ACTTS3 Encoding a Polyketide Synthase Is Essential for the Biosynthesis of ACT-Toxin and Pathogenicity in the Tangerine Pathotype of Alternaria alternata
}

\author{
Y. Miyamoto, ${ }^{1}$ A. Masunaka, ${ }^{1}$ T. Tsuge, ${ }^{2}$ M. Yamamoto, ${ }^{3}$ K. Ohtani, ${ }^{1}$ T. Fukumoto, ${ }^{1}$ K. Gomi, ${ }^{1}$ T. L. Peever, ${ }^{4}$ \\ Y. Tada, ${ }^{1}$ K. Ichimura, ${ }^{1}$ and K. Akimitsu ${ }^{1}$ \\ ${ }^{1}$ Faculty of Agriculture and Gene Research Center, Kagawa University, Miki, Kagawa 761-0795, Japan; ${ }^{2}$ Graduate School of \\ Bioagricultural Sciences, Nagoya University, Nagoya 464-8601, Japan; ${ }^{3}$ Faculty of Agriculture, Okayama University, \\ Okayama 700-8530, Japan; ${ }^{4}$ Department of Plant Pathology, Washington State University, Pullman 99164-6430, U.S.A.
}

Submitted 25 October 2009. Accepted 30 November 2009.

\begin{abstract}
The tangerine pathotype of Alternaria alternata produces host-selective ACT-toxin and causes Alternaria brown spot disease of tangerine and tangerine hybrids. Sequence analysis of a genomic BAC clone identified part of the $A C T$-toxin TOX (ACTT) gene cluster, and knockout experiments have implicated several open reading frames (ORF) contained within the cluster in the biosynthesis of ACT-toxin. One of the ORF, designated ACTTS3, encoding a putative polyketide synthase, was isolated by rapid amplification of cDNA ends and genomic/reverse transcription-polymerase chain reactions using the specific primers designed from the BAC sequences. The 7,374-bp ORF encodes a polyketide synthase with putative $\beta$-ketoacyl synthase, acyltransferase, methyltransferase, $\beta$-ketoacyl reductase, and phosphopantetheine attachment site domains. Genomic Southern blots demonstrated that $A C T T S 3$ is present on the smallest chromosome in the tangerine pathotype of $A$. alternata, and the presence of ACTTS3 is highly correlated with ACT-toxin production and pathogenicity. Targeted gene disruption of two copies of ACTTS3 led to a complete loss of ACT-toxin production and pathogenicity. These results indicate that ACTTS3 is an essential gene for ACT-toxin biosynthesis in the tangerine pathotype of A. alternata and is required for pathogenicity of this fungus.
\end{abstract}

Seven strains of Alternaria alternata (Fr.) Keissl. are known to produce host-selective toxins (HST) that are selectively toxic to certain cultivars of plants. HST of A. alternata are low molecular weight, secondary metabolites with toxicity toward distinct plant genotypes, and have the same specificity as infection by the toxin-producing pathogens (Nishimura and Kohmoto 1983; Scheffer and Livingston 1984; Walton 1996; Wolpert et al. 2003; Yoder 1980). These HST-producing strains are defined as seven distinct pathotypes of A. alternata (Kohmoto

Nucleotide sequence data for ACTTS3 is available in the DDBJ/GenBank database under accession number AB516321.

Current address of A. Masunaka: National Agricultural Research Center for Hokkaido Region, Hokkaido 082-0071, Japan.

Corresponding author: Kazuya Akimitsu; Telephone: +81-87-891-3131; Fax: +81-87-891-3021; E-mail: kazuya@ag.kagawa-u.ac.jp

* The $\boldsymbol{e}$-Xtra logo stands for "electronic extra" and indicates that two supplementary figures and one supplementary table are published online. Also, Figure 6 appears in color online. and Otani 1991; Nishimura and Kohmoto 1983) to emphasize the host specificity of each strain. Despite their close phylogenetic relationship (Kusaba and Tsuge 1994; 1995, Peever et al. 2004), each pathotype can be easily distinguished following inoculation on their respective host plants. Only a susceptible host can be damaged and reveal necrosis following inoculation of the pathotype as well as treatment with HST produced by the pathotype. No symptoms are visible on nonhosts following these treatments. The chemical structures of HST from six pathotypes of $A$. alternata have been elucidated (Kohmoto and Otani 1991), and HST biosynthetic genes were recently cloned from the Japanese pear pathotype, strawberry pathotype, tangerine pathotype, tomato pathotype, and apple pathotype of $A$. alternata (Akamatsu et al. 1997, 2003; Tanaka et al. 1999; Johnson et al. 2000; Masunaka et al. 2000, 2005; Tanaka and Tsuge 2000; Hatta et al. 2002; Ito et al. 2004; Ruswandi et al. 2005; Harimoto et al. 2007, 2008; Miyamoto et al. 2008, 2009).

The tangerine pathotype of A. alternata causes Alternaria brown spot disease, which affects many tangerine and mandarin cultivars and their hybrids, and the pathogenicity is dependent on the production of ACT-toxin (Kohmoto et al. 1979, 1991; Akimitsu et al. 2003). The structure of ACT-toxin is related to AK- and AF-toxins, which are the HST produced by the Japanese pear and strawberry pathotypes of A. alternata, and these toxins share a common 9,10-epoxy-8-hydroxy-9methyl-decatrienoic acid (decatrienoic acid) moiety in their structures (Nakashima et al. 1985, 1986; Kohmoto et al. 1993; Akimitsu et al. 2003) (Fig. 1). Homologues of the genes responsible for biosynthesis of the decatrienoic acid moiety were found in genomes from the tangerine, Japanese pear, and strawberry pathotypes (Tanaka et al. 1999; Masunaka et al. 2000, 2005; Tanaka and Tsuge 2000; Ruswandi et al. 2005; Miyamoto et al. 2008, 2009), and several genes in the tangerine pathotype identified and designated as $A C T$-toxin TOX (ACTT) 1, ACTT2, ACTT3, ACTTR, ACTT5, and ACTT6 (Masunaka et al. 2000, 2005; Miyamoto et al. 2008, 2009).

RNA silencing of multiple copies of ACTT2 encoding a putative hydrolase in the genome of tangerine pathotype led to a complete loss of ACTT2 transcripts and ACT-toxin production as well as pathogenicity (Miyamoto et al. 2008). ACTT5 encoding a putative acyl-CoA synthetase and ACTT6 encoding a putative enoyl-CoA hydratase were also identified by sequencing of cosmid clone containing open reading frames (ORF) of these genes together with ACTT2, ACTT3, and ACTTR (Miyamoto et al. 2009). Targeted gene disruption of two copies of ACTT5 significantly reduced ACT-toxin pro- 
duction and virulence, and targeted gene disruption of two copies of ACTT6 led to a complete loss of ACT-toxin production and pathogenicity (Miyamoto et al. 2009).

Using partial regions of a chromosomal DNA sequence from a BAC clone selected by the ACTTR probe, we identified several ORF of the tangerine pathotype, isolate $\mathrm{SH} 20$, and we report here the distribution and role in ACT-toxin production and pathogenicity of one of these ORF encoding a putative polyketide synthase (PKS) designated as ACTTS3.

\section{RESULTS}

Sequence identification and characterization of ACTTS3 in isolate $\mathrm{SH} 20$ of the tangerine pathotype of $A$. alternata.

BAC clone $2 \mathrm{H} 2$ was sequenced to $5 \times$ redundancy by shotgun sequencing using a capillary DNA sequencer. Sequence analysis identified several ORF of known ACTTS genes, including ACTT1, ACTT2, ACTT3, ACTTR, ACTT5, and ACTT6, and additional ORF, including ACTTS1 encoding a putative nonribosomal peptide synthetase (NRPS), ACTTS 2 encoding a putative enoyl-reductase, ACTTS4 encoding a putative NRPS, some additional ORF of unknown function, multiple transposon-like sequences, and a putative PKS.

Using the genomic sequence of the ORF encoding the putative PKS in this BAC clone, we designed several pairs of genespecific primers for reverse-transcription polymerase chain reaction (RT-PCR) and $5^{\prime}$ and $3^{\prime}$ rapid amplification of cDNA ends (RACE) to identify the entire transcript (Table 1). The PKS gene designated ACTTS3 (accession no. AB516321) is $7,374 \mathrm{bp}$ in nucleotide length and predicted to encode a protein of 2,457 amino acids. The promoter region of ACTTS3 has a GATA consensus motif (-50), a TATC motif (-339), potential binding sites for the nitrogen-induced AreA (Marzluf 1993), an ATTG motif (-324) of the light-regulated WC1/ WC2 (Linden and Macino 1997), transcriptional activators, and C/EBP (camp-activated protein) (Rangan et al. 1996) (data not shown). FASTA searches using the sequence of the $\beta$-ketoacyl synthase domain indicated that ACTTS3 had sequence similarity to fungal PKS. ACTTS3 had a significant match to the 6-methylsalicylic acid synthase gene (6MSAS; X55776) from Penicillium patulum (Beck et al. 1990) and pksL2 of Aspergillus parasiticus (APU52151) (Feng and Leonard 1995). This latter gene is involved in the biosynthesis of aflatoxin and is classified through phylogenic analysis as a fungal type I PKS nested within a large clade comprising all bacterial type I PKS (Kroken et al. 2003; Moss et al. 2004) (Fig. 2; Supplementary Fig. 1). Functional domain searches with ACTTS3 using the Pfam program (Finn et al. 2008) identified five catalytic domains arranged in order from the $\mathrm{N}$ terminal $\beta$-ketoacyl synthase (KS; 6 to 385), acyltransferase (AT; 549 to 889), methyltansferase (ME; 1,422 to 1,525), $\beta$ ketoacyl reductase (KR; 2,089 to 2,265 ), and phosphopantetheine attachment site (PP; 2,381 to 2,447) (Fig. 2; Supplementary Fig. 2). The organization of PKS functional domains in ACTTS3 is similar to that of fungal reducing PKS, such as PKS4 and PKS5 of Botryotinia fuckeliana (Kroken et al. 2003), PKS16 of Cochliobolus heterostrophus (Kroken et al. 2003), and lvB of A. terreus (Kennedy et al. 1999) (Fig. 2).

The distribution of ACTTS3 in the genome of seven pathotypes and nonpathogenic isolates of Alternaria alternata was examined by genomic Southern blotting. The ACTTS3 probe hybridized only to genomes of tangerine pathotype isolates producing ACT-toxin (Fig. 3A). The ACTTS3 probe hybridized the genomes of tangerine pathotype isolates from Israel, Australia, and Colombia (Fig. 3B), indicating that ACTTS3 is distributed among all worldwide phylogenetic lineages of the pathogen. Southern blots of pulsed-field gel electrophoresis (PFGE) gels demonstrated that ACTTS3 located to the same small chromosome that was previously shown to carry other ACTT genes among Florida tangerine pathotype isolates (Masunaka et al. 2005; Miyamoto et al. 2008, 2009) (Fig. 3C).

The distribution of ACTTS3 copies in the genome of tangerine pathotype isolate $\mathrm{SH} 20$ was further examined by genomic Southern blotting using multiple restriction enzymes. The ACTTS3 probe detected one fragment when genomic DNA was digested with EcoRV, PstI, HindIII, and BglII, which have no predicted restriction sites within the region of sequence made for the probe (Fig. 4B). The expected two bands were observed upon digestion with BanIII, which has one restriction site predicted within the ACTTS3 probe sequence. Expected sizes of bands matching with the sequence-based map (Fig. 4B) are shown with examples of a 3.0-kb fragment digested with HindIII (Fig. 4A and B) and a 1.2-kb fragment digested with SacI, which had one predicted restriction site within the probe sequence (Fig. 4A and B); however, only one band was observed upon digestion with the enzyme, as expected due to the location of the site within the probe region; it was placed at the edge of the sequence and the shorter fragment was not long enough to detect by the probe. $X b a \mathrm{I}$ also has one site at the edge within the ACTTS3 probe sequence, similar to the case of SacI digestion, and only a single band was observed, as expected.

\section{Targeted gene disruption of ACTTS3}

for functional analysis of ACT-toxin biosynthesis.

To examine the role of ACTTS3 in ACT-toxin production and pathogenicity, targeting vector ACTTS3hph was constructed. The vector contained two partial regions of ACTTS3

\section{ACT-toxin}

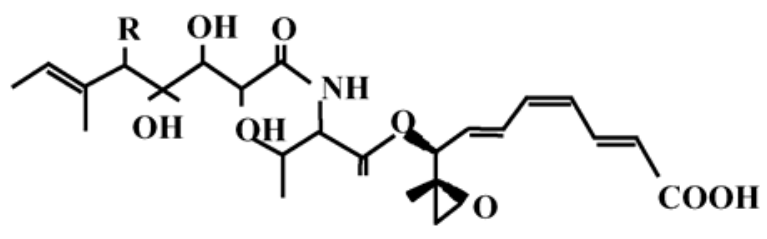
ACT-toxin I: $\mathbf{R}=\mathbf{O H}$
ACT-toxin II: $\mathbf{R}=\mathbf{H}$

AK-toxin

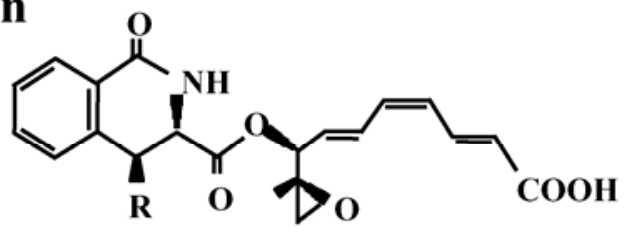

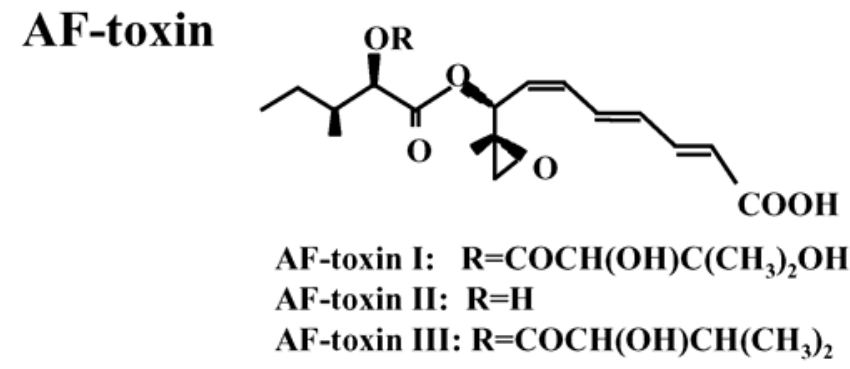

Fig. 1. Chemical structures of ACT-, AK-, and AF-toxins produced by the tangerine pathotype, Japanese pear pathotype, and strawberry pathotype of Alternaria alternata, respectively (Nakashima et al. 1985, 1986; Kohmoto et al. 1993). 
with an $h p h$ cassette (Fig. 5A) which was used for ACTTS3 disruption mediated by homologous recombination. Screening for reduced ACT-toxin production and Southern hybridization identified transformant rep14-2, which showed a reduction in the toxicity in culture filtrates, and a shift in the 10-kb ACTTS3 band present in the wild type to a $13.0-\mathrm{kb}$ band in the transformant, indicating insertion of the vector construct at the target site (Fig. 5A and C). The $h p h$ probe hybridized only to the 13.0-kb-shifted band of transformant rep14-2 (data not shown) and the $10-\mathrm{kb}$ band shown in the wild type still remained in rep14-2 (Fig. 5C), suggesting that ACTTS3 exists in more than one copy in the genome of $\mathrm{SH} 20$ and the vector construct was inserted in only one of the multiple copies. Transcription analysis of ACTTS3 by RT-PCR with the primer set of PKS1F3 and PKS1R3 generated an amplicon of predicted size (966 bp) from total RNAs of both the wild type and transformant rep14-2, and the product hybridized to the ACTTS3 probe (Fig. 6A). Intensity of the band was reduced in the transformant rep14-2 compared with that of the wild type (Fig. 6A), and the PCR reaction without addition of reverse transcriptase did not amplify any product. The actin gene, which is known to be expressed constitutively, was amplified equally to that observed for the wild type (Fig. 6A). ACT-toxin production in culture filtrates seeded with either the wild type or transformant rep14-2 were compared by high-performance liquid chromatography (HPLC). A $61 \%$ reduction in ACT-toxin peak area was observed in transformant rep14-2 (Fig. 6B). When spores of transformant rep14-2 were spray inoculated on the lower leaf surfaces of susceptible cv. Iyokan, typical leaf spots appeared but disease severity was reduced significantly compared with inoculation with the wild type (Fig. 6C).

To disrupt the second copy of ACTTS3, the PCR fragment corresponding to a partial region of ACTTS3 was amplified using the primer set PKS1F12NotI and PKS1R11BamHI and subcloned into the NotI and BamHI sites of transformation

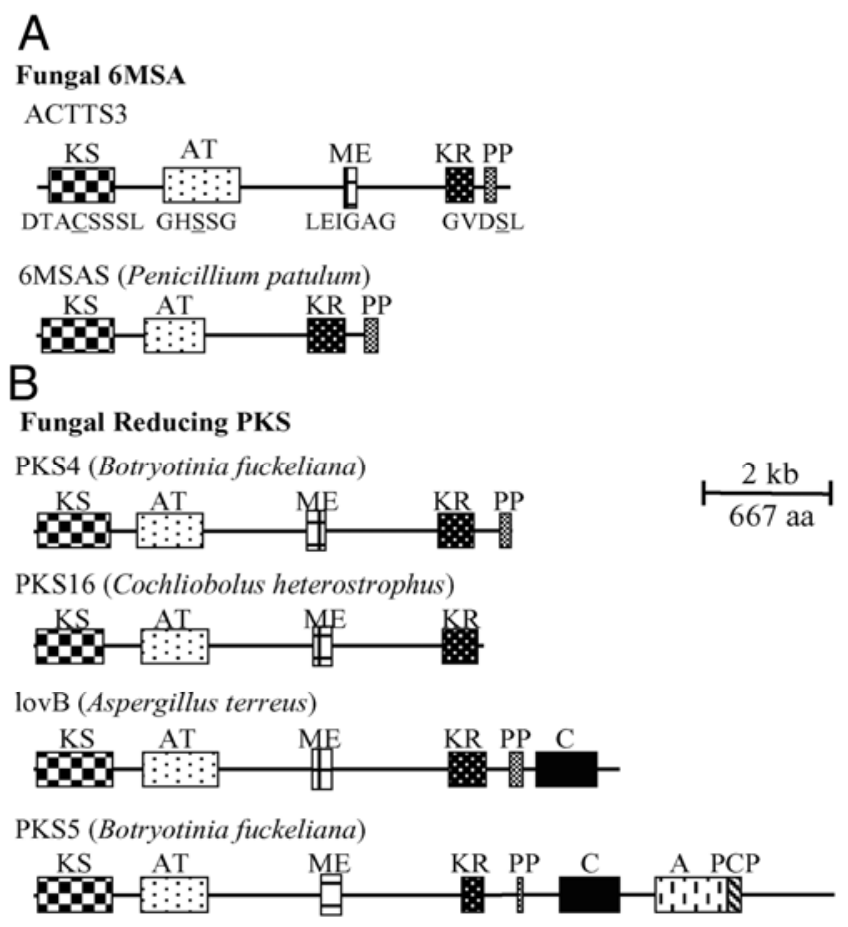

Fig. 2. Organization of catalytic domains in ACTTS3 and several fungal polyketide synthase (PKS) genes. Comparison of domain organization of ACTTS3 with A, fungal 6MSA and $\mathbf{B}$, fungal reducing PKS based on a genealogy of the $\beta$-ketoacyl synthase (KS) domain by Kroken and associates (2003). Catalytic domains: $\mathrm{AT}=$ acyltransferase, $\mathrm{ME}=$ methyl-transferase, $\mathrm{KR}=\beta$-ketoreductase, $\mathrm{PP}=$ peptidyl carrier domain, $\mathrm{C}=$ condensation, and $\mathrm{A}=$ AMP-binding. Accession numbers for these PKS are X55776 for 6MSAS (Penicillium patulum), AY495609 for PKS4 (Botryotinia fuckeliana), AY495657 for PKS16 (Cochliobolus heterostrophus), AF151722 for lovB (Aspergillus terreus), and AY495610 for PKS5 (Botryotinia fuckeliana).

Table 1. Sequences of gene-specific primers used for this study

\begin{tabular}{|c|c|c|c|}
\hline Primer name & Primer sequences $\left(5^{\prime}-3^{\prime}\right)$ & Purpose $^{a}$ & Reference \\
\hline ACTTRF1 & CAACAGCAGTCGAACC & ACTTR probe & Miyamoto et al. 2008 \\
\hline ACTTRR1 & CGTCTAGAACGAATCG & ACTTR probe & Miyamoto et al. 2008 \\
\hline PKS1F3 & GCTGACGAGACTGTTG & RT-PCR for ACTTS3 & This study \\
\hline PKS1R3 & CTGTGTTTCCCCACAG & RT-PCR for ACTTS3 & This study \\
\hline actinFor & AGATGACACAGATTGTTTTCGAGACC & RT-PCR for actin gene & Miyamoto et al. 2009 \\
\hline actinRev & AGTTGAAAGTGGTGACGTGGATACC & RT-PCR for actin gene & Miyamoto et al. 2009 \\
\hline PKS1F1 & TGGATCCTGCTCAACG & RT-PCR for ACTTS3 & This study \\
\hline PKS1R1 & ATTGGCGGGTATGGTG & RT-PCR for ACTTS3 & This study \\
\hline $2 \mathrm{H} 2 \mathrm{C} 27 \mathrm{R} 1$ & GATGTGCAGGCCTAGCA & RT-PCR for ACTTS3 & This study \\
\hline $2 \mathrm{H} 2 \mathrm{C} 27 \mathrm{~F} 1$ & AGATGCAGTCCGACCG & RT-PCR for ACTTS3 & This study \\
\hline PKS1F2 & TGCGCCGAACCTTATC & RT-PCR for ACTTS3 & This study \\
\hline PKS1R2 & TGGCGGCAACTTCACA & RT-PCR for ACTTS3 & This study \\
\hline $\mathrm{C} 27 \mathrm{Fe} 2$ & CAACGTGCGTGTA & RT-PCR for ACTTS3 & This study \\
\hline $\mathrm{C} 27 \mathrm{Rm} 2$ & TTCGAGCTCAACATCCAGGT & RT-PCR for ACTTS3 & This study \\
\hline $\mathrm{C} 26 \mathrm{Rm}$ & GCTTTGTATCGTATGCC & RT-PCR for ACTTS3 & This study \\
\hline $2 \mathrm{H} 2 \mathrm{C} 26 \mathrm{~F} 1$ & TTAGTCAGGCGGTCGA & RT-PCR for ACTTS3 & This study \\
\hline $2 \mathrm{H} 2 \mathrm{C} 26 \mathrm{R} 1$ & TGCAGCAAGTCGGACT & RT-PCR for ACTTS3 & This study \\
\hline PKS1F4 & GACACCGCGAATCATG & RT-PCR for ACTTS3 & This study \\
\hline PKS1R4 & CTGGCAACGTACCCAA & RT-PCR for ACTTS3 & This study \\
\hline PKS1R6 & CGCGCATCTGCAGATCCCACCAATCGGA & 5' RACE for ACTTS3 & This study \\
\hline PKS1R5 & CAACAAACACAGACGTGTGGGTACCCTGC & 5' RACE for ACTTS3 & This study \\
\hline PKS1F5 & GGTGGTCAATCCAATTACCACG & 3' RACE for ACTTS3 & This study \\
\hline PKS1F6 & CGTGCTCGAGGTCTTGCTGCATCGGTCA & 3' RACE for ACTTS3 & This study \\
\hline PKS1F10HindIII & CCCAAGCTTGGGTGTGAAGTTGCCGCCA & ACTTS3hph construct & This study \\
\hline PKS1R3EcoRV & GATATCCTGTGTTTCCCCACAG & ACTTS3hph construct & This study \\
\hline PKS1F11BamHI & CGGGATCCCGTGTGGTTGACCAAGGG & ACTTS3hph construct & This study \\
\hline PKS1R10KpnI & GGGGTACCCCGACATTTGCGGTCGCA & ACTTS3hph construct & This study \\
\hline PKS1F12NotI & GCGGCCGCCCAGACTGTCTGGGA & ACTTS3NPTII construct & This study \\
\hline PKS1R11BamHI & CGGGATCCCGCAAGTAGCCACCAGGT & ACTTS3NPTII construct & This study \\
\hline NPTIIF1EcoRI & GGAATTCCATGATTGAACAAGATGGATTGC & ACTTS3NPTII construct & This study \\
\hline NPTIIR1EcoRI & GGAATTCCTCAGAAGAACTCGTCAAGAA & ACTTS3NPTII construct & This study \\
\hline
\end{tabular}

${ }^{\mathrm{a}} \mathrm{RACE}=$ rapid amplification of cDNA ends and RT-PCR $=$ reverse-transcription polymerase chain reaction. 
vector pAGY carrying the NPTII cassette to generate ACTTS3NPTII (Fig. 5B). The single-copy disruptant rep14-2 was retransformed with the ACTTS3NPTII vector, and the double mutants which showed resistance to both hygromycin and geneticin were selected. Culture filtrates from one of the transformants (D1-2) did not show any toxicity to Iyokan. Integration of ACTTS3NPTII at the target site was analyzed by Southern blotting and transformant D1-2 showed the expected bands of 13.0 and $20.0 \mathrm{~kb}$, whereas the native $10.0-\mathrm{kb}$ band was observed in the wild type (Fig. 5C). No ACTTS3 transcript was detected by RT-PCR in total RNA from transformant D1-2 (Fig. 6A). HPLC analysis also did not detect any trace of ACT-toxin in the culture filtrates of transformant D1-2 (Fig. 6B), and inoculation of spores from transformant D1-2 on the Iyokan leaves were nonpathogenic (Fig. 6C). No apparent differences in growth (Fig. 6D), sporulation, and spore germination in vitro were detected among the wild type, single- copy disruptant rep14-2, and double-copy disruptant D1-2 (data not shown).

\section{DISCUSSION}

Several ORF responsible for ACT-toxin biosynthesis have been identified in the tangerine pathotype of A. alternata, and these ORF are clustered on a $1.9-\mathrm{Mb}$ chromosome in reference isolate SH20 (Masunaka et al. 2000, 2005; Miyamoto et al. 2008, 2009). In the current study, we have identified a 7,374bp ORF encoding a putative PKS and named it ACTTS3. We determined the distribution of ACTTS 3 among different pathogenic and nonpathogenic isolates of A. alternata and provided biochemical and molecular genetic evidence for the involvement of ACTTS3 in ACT-toxin production and pathogenicity.

FASTA searches using the KS domain sequence of ACTTS3 revealed that ACTTS3 belongs to the fungal type I PKS family

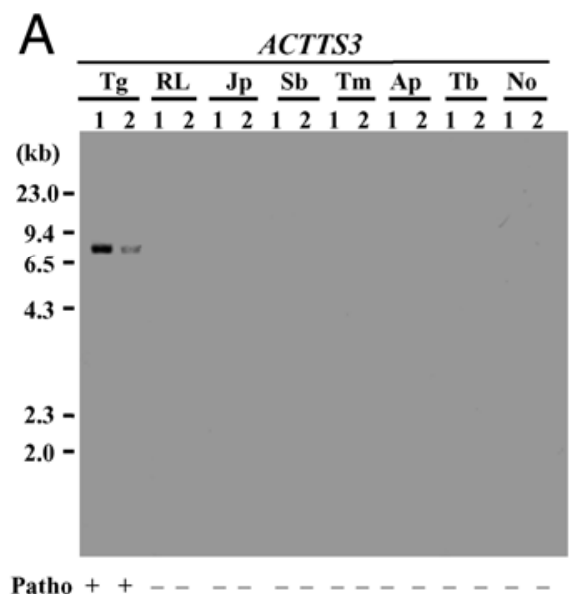

B

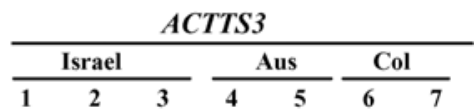

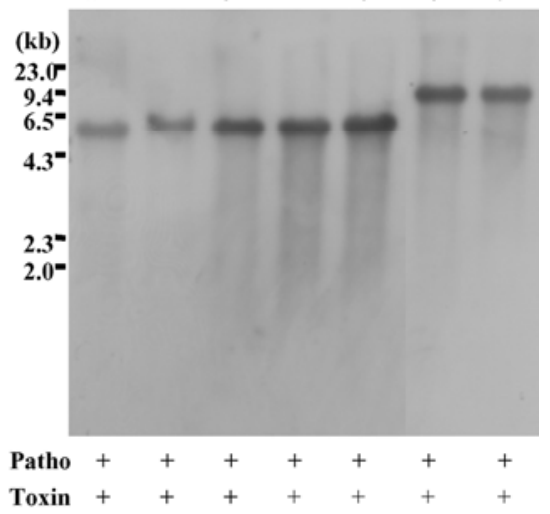

Patho ++----------
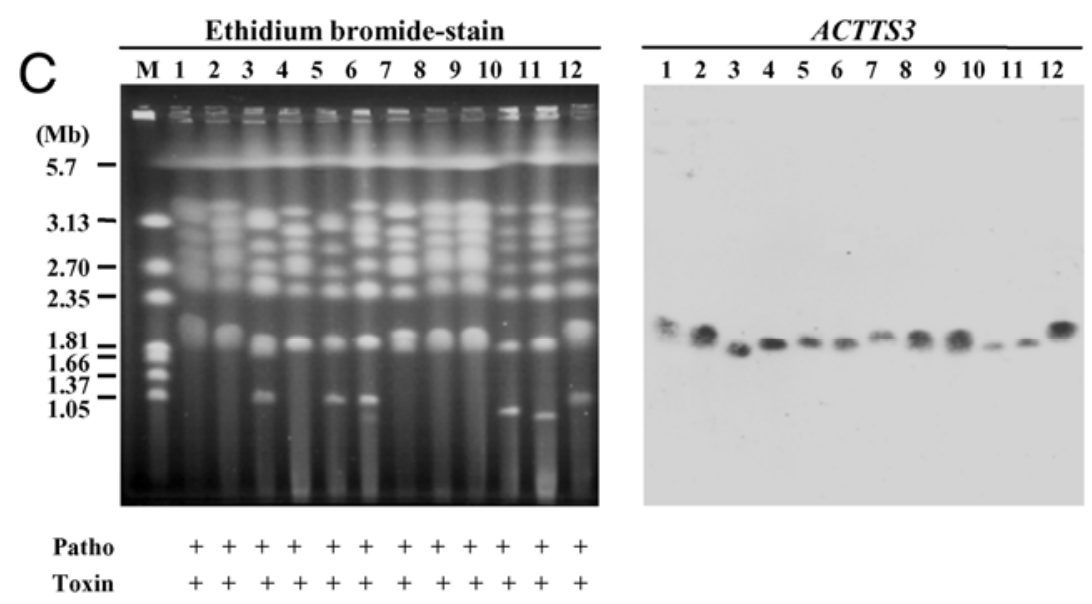

Fig. 3. Distribution of ACTTS3 in the genomes of different pathogenic and nonpathogenic isolates of Alternaria alternata. A, Genomic Southern blot analysis of ACTTS3 hybridizing to seven distinct host-selective toxin-producing pathotypes and non-pathogenic strains of A. alternata. Abbreviations used are: Tg, tangerine pathotype, isolates SH11 (lane 1) and SH20 (lane 2); RL, rough lemon pathotype, isolates HC1 (lane 1) and HC8 (lane 2); JP, Japanese pear pathotype, isolates G31(1) and G90-A2 (2), Sb; strawberry pathotype, isolates NAF-3 (lane 1) and NAF-8 (lane 2); Tm, tomato pathotype, isolates 91080804 (lane 1) and AL-4 (lane 2); Ap, apple pathotype, isolates AM-1 (1) and O-159 (2); Tb, Tobacco pathotype, isolates No. 122-2 (lane 1) and AT204 (lane 2); and No, nonpathogenic isolates O-94 (lane 1) and HC14 (lane 2). B, Genomic Southern blot analysis of the distribution of ACTTS3 among a worldwide sample of tangerine pathotype isolates of A. alternata. Genomic DNA was digested with EcoRV. Isolates used were 45-001 (lane 1), 45-003 (lane 2), and 45-013 (lane 3) from Israel; Aus-1 (lane 4) and Aus-9 (lane 5) from Australia; and 45-014 (lane 6) and 11-CEN-S (lane 7) from Colombia. Sizes of marker DNA fragments (HindIII-digested $\lambda$ DNA) are indicated at left in both panels. C, Chromosomal location of ACTTS3 among tangerine pathotype isolates from citrus in Florida. Chromosomal DNAs of each isolate were separated using pulsed-field gel electrophoresis in 0.8\% agarose gel (left panel). Chromosomes were transferred to nylon membrane and detected with digoxigenin-labeled ACTTS3 probe (right panel). Hansenula wingei chromosome size markers are indicated at left. Isolates examined were from two A. alternata populations separated by approximately $20 \mathrm{~km}(\mathrm{SH}=\mathrm{Shinn} \mathrm{Grove}$ and EV = Evenhouse Rd.) (Peever et al. 1999) in order from left to right: SH11, SH12, SH13, SH14, SH15, SH18, SH19, SH20, SH21, EV10, EV13, and EV14. Pathogenicity to Iyokan leaves (Patho) and ACT-toxin production (Toxin) are indicated at bottom of figure. 
(Moss et al. 2004). This family includes the 6MSAS gene of $P$. patulum (Beck et al. 1990) which was nested within a large clade comprising all bacterial type I PKS genes (Kroken et al. 2003). Classifying PKS genes by domain organization in addition to sequence similarity within domains is a more effective way to categorize PKS genes because genes encoding fungal PKS generally consist of large, multifunctional ORF. Topologies of phylogenies estimated from different regions of these ORF can be incongruent (Kroken et al. 2003; Moss et al. 2004). Indeed, the organization of PKS functional domains in ACTTS3 consisting of KS-AT-ME-KR-PP suggests that it may be more similar to the organization of fungal reducing PKS, which have lost the DH domain but gained the ME domain. An example of this is PKS16 of C. heterostrophus (Kroken et al. 2003), which synthesizes a precursor of T-toxin, one of the HST produced by Cochliobolus spp. (Yang et al. 1996). These reduced polyketides are frequently synthesized from CoA thioesterified carboxylic acids rather than acetyl and malonyl $\mathrm{CoA}$, and the extent of keto-group processing varies from one condensation cycle to the next (Hopwood 1997). In addition, one or more sites may be methylated by a ME domain (Abe et al. 2002), although many of the predicted PKS in the PKS clade that produces reduced PKS have become highly divergent and some contain a presumably nonfunctional ME domain (Kroken et al. 2003).

Genes required for the synthesis of Alternaria spp. HST, such as the $A K$-toxin TOX $(A K T)$ cluster controlling AK-toxin pro-

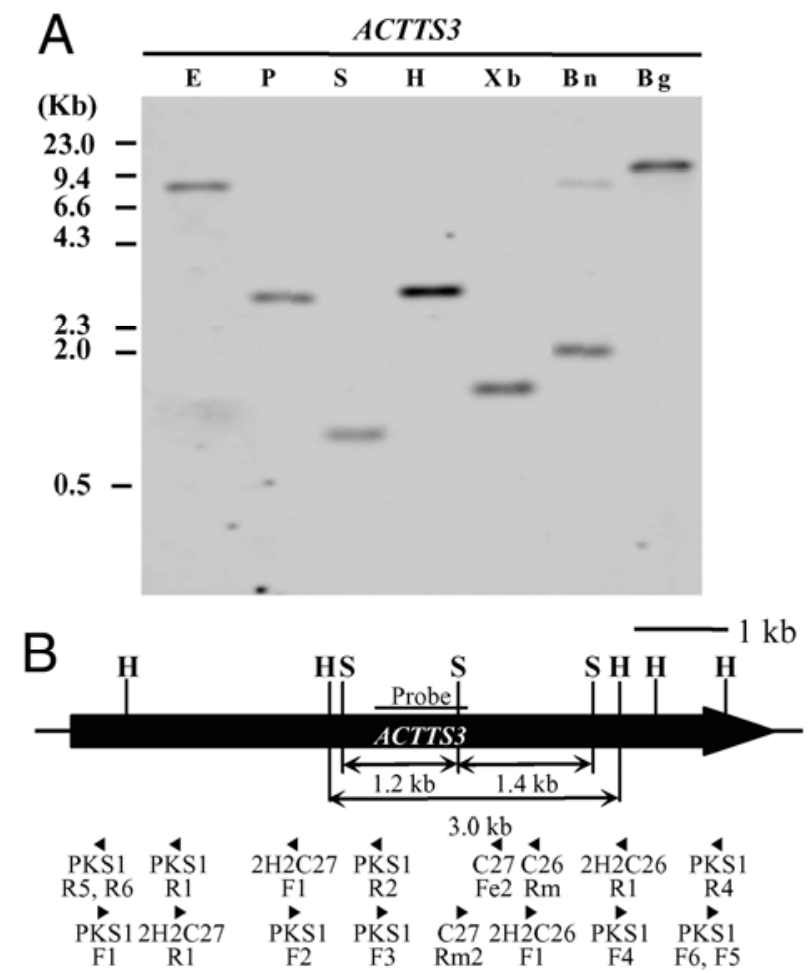

Fig. 4. Estimation of copy number of ACTTS3 in the genome of the tangerine pathotype of Alternaria alternata. A, Genomic Southern blot analysis of ACTTS3 in isolate $\mathrm{SH} 20$ of the tangerine pathotype of A. alternata digested with several different restriction enzymes. DNA of isolate $\mathrm{SH} 20$ was digested with EcoRV (lane E), PstI (lane P), SacI (lane S), HindIII (lane $\mathrm{H}$ ), XbaI (lane $\mathrm{Xb}$ ), BanIII (lane $\mathrm{Bn}$ ), and BglII (lane $\mathrm{Bg}$ ), and the digested DNA was transferred to nylon membrane probed with a digoxigenin-labeled ACTTS3 probe. Sizes of marker DNA fragments (HindIIIdigested $\lambda$ DNA) are indicated at left. B, Restriction and primer annealing site map of ACTTS3 open reading frame and flanking regions. Abbreviations $\mathrm{H}$ and $\mathrm{S}$ indicate HindIII and SacI restriction sites, respectively. Arrows indicate location of oligonucleotide primers used in polymerase chain reaction. Scale bar indicates $1 \mathrm{~kb}$. duction (Tanaka et al. 1999) and the AF-toxin TOX (AFT) cluster controlling AF-toxin production (Hatta et al. 2002), are tightly clustered. Genes responsible for ACT-toxin production (ACTT1, ACTT2, ACTT3, ACTTR, ACTT5, and ACTT6 and ACTTS1, ACTTS2, ACTTS3, and ACTTS4) are also clustered, and this cluster has been designated as the ACTT. These genes are located on a single chromosome of 1.9 to $2.0 \mathrm{Mb}$ in ACT-toxinproducing isolates from Florida (Masunaka et al. 2000, 2005; Miyamoto et al. 2008, 2009). Generally, only A. alternata strains producing HST are known to carry chromosomes of less than $2.0 \mathrm{Mb}$ and these chromosomes appear to be completely absent from nonpathogenic strains (Akamatsu et al. 1999; Masunaka et al. 2000, 2005; Hatta et al. 2002). The small chromosomes carrying toxin genes do not appear to carry genes essential for normal growth and reproduction. Loss of these chromosomes has no obvious phenotypic effect on in vitro growth, resulting in these chromosomes being called conditionally dispensable (Covert 1998; VanEtten et al. 1998; Hatta et al. 2002). Interestingly, gene clusters responsible for the biosynthesis of HST, such as the $A K T, A F T, A M T, A L L T$, and ACTT clusters in the Japanese pear, strawberry, apple, tomato, and tangerine pathotypes, respectively, are all located on small, dispensable chromosomes (Akamatsu et al. 1997, 2003; Tanaka et al. 1999; Masunaka et al. 2000, 2005; Tanaka and Tsuge 2000; Harimoto et al. 2007, 2008; Miyamoto et al. 2008, 2009). The AKT, AFT, and ACTT clusters share homologous coding and noncoding regions, some of which are responsible for the production of the common decatrienoic acid moiety in the structures of AK-, AF-, and ACT-toxins (Tanaka et al. 1999; Masunaka et al. 2000; Tanaka and Tsuge 2000; Hatta et al. 2002; Ito et al. 2004; Ruswandi et al. 2005; Miyamoto et al. 2008, 2009).

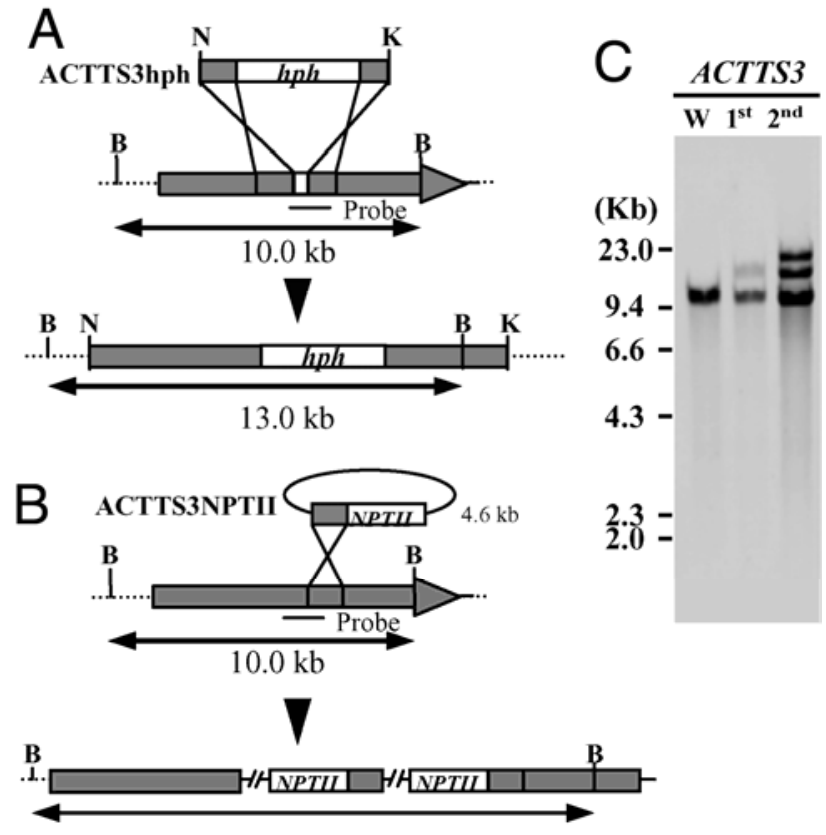

$20.0 \mathrm{~kb}$

Fig. 5. Targeted gene disruption of ACTTS3 in the tangerine pathotype of Alternaria alternata. A, Disruption construct ACTTS3hph and predicted integration pattern in single-copy disruptant rep14-2 of tangerine pathotype isolate $\mathrm{SH} 20 . \mathrm{N}, \mathrm{K}$, and B indicate NotI, KpnI, and BglII restriction sites, respectively. B, Disruption construct ACTTS3NPTII and predicted integration pattern in double-copy disruptant D1-2. B indicates a $B g l \mathrm{II}$ restriction site. C, Genomic Southern blot of ACTTS3 hybridizing to the wild type (lane W), single-copy disruptant rep14-2 (1st), and double-copy disruptant D1-2 (2nd). Genomic DNA was digested with $B g l \mathrm{II}$ and fractionated in $0.8 \%$ agarose following hybridization with digoxigenin-labeled ACTTS 3 probe. Sizes of marker DNA fragments (HindIII-digested $\lambda$ DNA) are indicated at left. 
ACTTS3 only hybridized to tangerine pathotype genomes and not to the genomes of seven noncitrus pathotypes and nonpathogenic isolates of $A$. alternata, indicating that ACTTS3 is unique to the tangerine pathotype. ACTTS3 was found in tangerine pathotype isolates from Australia, Israel, and Colombia but did not hybridize to the genomes of the Japanese pear pathotype or strawberry pathotype isolates. This suggests that ACTTS3 is not required for the biosynthesis of decatrienoic acid, a common moiety in ACT-, AK-, and AF-toxins, but, rather, is required for synthesis of some unique component of ACT-toxin. ACT-toxin consists of three parts, including decatrienoic acid, valine (amino acid), and a polyketide moiety (Fig. 1). Because the 2,457 amino acid sequence of ACTTS3 shows a strong sequence similarity to a PKS, ACTTS 3 is predicted to be responsible for the biosynthesis of the polyketide moiety of ACT-toxin. ACTTS3 was found only on the smallest chromosomes in the genomes $(1.9$ to $2.0 \mathrm{Mb})$ of all tangerine pathotype isolates obtained in Florida citrus fields, and the presence of this gene is correlated with ACT-toxin production and pathogenicity to Iyokan tangerine.

To definitively test the hypothesis that ACTTS3 is required for biosynthesis of the polyketide moiety of ACT-toxin, we generated single- and double-copy disruptants of ACTTS3 using gene replacement and targeted gene disruption mediated by homologous recombination. The single-copy mutant rep142 showed reductions in transcript accumulation of ACTTS3, ACT-toxin production, and pathogenicity, and the double-copy disruptant D1-2 showed a complete loss of ACTTS3 transcription as well as ACT-toxin production and pathogenicity. Genomic Southern blots of the double-copy mutant probed with the ACTTS3 probe revealed a band of equal size to that observed in the wild type, which might indicate additional copies of ACTTS3 in the genome of D1-2. However, because there was no trace of ACTTS3 transcript and ACT-toxin production detected in the double-copy disruptant D1-2, the band likely represents a pseudogene with defects in the promoter region, resulting in transcriptional dysfunction. Lack of ACT-toxin production was consistently associated with a loss of pathogenicity which has been consistently demonstrated in several independent studies of HST (Akamatsu et al. 1997, 2003; Tanaka et al. 1999; Johnson et al. 2000; Masunaka et al. 2000, 2005; Tanaka and Tsuge 2000; Hatta et al. 2002; Ito et al. 2004; Ruswandi et al. 2005; Harimoto et al. 2007, 2008; Miyamoto et al. 2008, 2009). We conclude that ACTTS3 is critically required for ACT-toxin biosynthesis and is essential for pathogenicity of the tangerine pathotype of A. alternata. We speculate that the function of ACTTS3 is to elongate the polyketide chain portion of ACT-toxin that is unique to this toxin.

\section{MATERIALS AND METHODS}

\section{Fungal strains and plants.}

Young leaves (midrib length: 2 to $4 \mathrm{~cm}$ ) were obtained from the citrus cv. Iyokan tangor (Citrus iyo hort. Ex Tanaka) grown in the greenhouse and used for inoculation and toxin sensitivity assays as described previously (Masunaka et al. 2000; Miyamoto et al. 2008, 2009). Iyokan is susceptible to the tangerine pathotype of A. alternata and sensitive to ACT-toxin (Kohmoto et al. 1991). Isolates of A. alternata tangerine pathotype were previously sampled from leaf lesions of Minneola tangelo ( $C$. reticulata Blanco $\times C$. paradisi Macf.) in Florida for studies of the population structure and the mechanism of host specificity of the Alternaria brown spot pathogen (Peever et al. 1999; Masunaka et al. 2000, 2005; Miyamoto et al. 2008, 2009) and listed in Supplementary Table S1. Isolates SH11, SH12, SH13, SH14, SH15, SH18, SH19, SH20, and
SH21 and EV10, EV13, and EV14 of the tangerine pathotype and isolates $\mathrm{HC} 1$ and $\mathrm{HC} 8$ of the rough lemon pathotype were isolated from leaf lesions on both hosts in central Florida (Peever et al. 1999; Masunaka et al. 2000, 2005). Isolate SH20 has been used as the reference isolate for the brown spot pathogen in several previous studies (Masunaka et al. 2000, 2005; Miyamoto et al. 2008, 2009). Isolate BC3-5-1-OS2A, a dual pathotype isolate producing both ACT-toxin and ACR-toxin (Masunaka et al. 2005), was isolated from a rough lemon leaf lesion in central Florida. Isolates 45-001, 45-003, and 45-013 from Israel; Aus-1 and 9 from Australia; and 45-014 and 11CEN-S from Colombia were also used (Peever et al. 1999). Laboratory stocks (Ito et al. 2004; Miyamoto et al. 2009) of Japanese pear pathotype isolates G31 and G90-A2, strawberry pathotype isolates NAF-3 and NAF-8, tomato pathotype isolates 91080804 and AL-4, apple pathotype isolates AM-1 and O-159, and tobacco pathotype isolates 122-2 and AT-204 were included for comparative purposes. Nonpathogenic isolates HC14 from rough lemon (Peever et al. 1999; Masunaka et al. 2000) and A. alternata isolate O-94, obtained from H. Otani, Tottori University, Japan, were included as nonpathogenic controls. All isolates, including transformants of isolate $\mathrm{SH} 20$, were stored in $25 \%(\mathrm{vol} / \mathrm{vol})$ glycerol at $-85^{\circ} \mathrm{C}$ and cultures
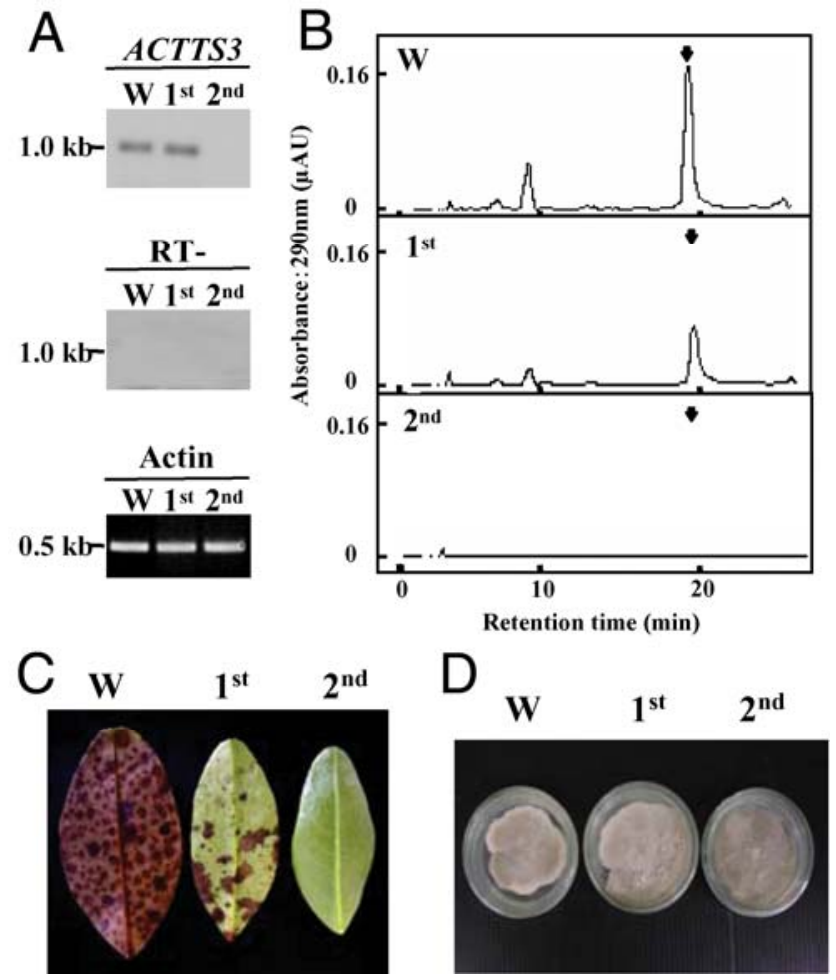

Fig. 6. ACT-toxin production and pathogenicity of ACTTS3 disruptants. A, reverse-transcription polymerase chain reaction (RT-PCR) and Southern blot analysis of ACTTS3 transcription in disruptants. Top panel: RT-PCR products of ACTTS3 from total RNA of the wild type (lane W), singlecopy disruptant rep14-2 (1st), and double-copy disruptant D1-2 (2nd) hybridized with digoxigenin-labeled ACTTS3 probe. Middle panel: RT- indicates negative controls without addition of reverse transcriptase. Lower panel: Amplification of actin gene was used as positive control for RTPCR system as well as to test RNA loading. B. High-performance liquid chromatography charts of each extract from culture filtrates of ACTTS3 disruptants. Arrows indicate peaks for ACT-toxin for the wild type (lane W), single-copy disruptant rep14-2 (1st), and double-copy disruptant D1-2 (2nd). C, Symptoms caused by inoculation of Iyokan leaves with the wild type (lane W), single-copy disruptant rep14-2 (1st), or double-copy disruptant D1-2 (2nd) $48 \mathrm{~h}$ after inoculation. D, Morphology in culture on potato dextrose agar of the wild type (lane W), single-copy disruptant rep14-2 (1st), and double-copy disruptant D1-2 (2nd). 
were initiated from the stocks for each experiment by growth on V8 juice agar or potato dextrose agar (PDA) plates.

\section{Nucleic acid manipulations.}

Extraction of genomic DNA and total RNA, genomic PCR, RT-PCR, RACE, and Southern blot hybridization using digoxigenin (DIG)-PCR-labeled probes (Roche, Basel, Switzerland) were carried out as previously described (Isshiki et al. 2001; Masunaka et al. 2000, 2005; Katoh et al. 2006, 2007; Miyamoto et al. 2008, 2009). Restriction enzyme digestion of genomic DNA $(5 \mu \mathrm{g})$, agarose gel electrophoresis, and capillary transfer were conducted under standard conditions (Sambrook and Russell 2001). A BAC library was constructed using A. alternata isolate BC3-5-1-OS2A as described previously (Masunaka et al. 2005; Hatta et al. 2006). Genomic DNA fragments of approximately $100 \mathrm{~kb}$ from BC-3-5-1-OS2A were ligated into the BamHI site of pcc1 BACTM vector (Epicentre, Madison, WI, U.S.A.) (Masunaka et al. 2005; Miyamoto et al. 2008). The ligated vector was introduced into Escherichia coli (DH10B) (Invitrogen, San Diego, CA, U.S.A.) by electroporation. After checking the titer of the transformation, colonies screened by blue-white selection were randomly picked into 384-well plates (total of 9 plates) as a glycerol stock of the library. The genomic BAC library was screened by colony hybridization with a DIG-PCR-labeled ACTTR probe using the primer set ACTTRF1 (5'-CAACAGCAGTCGAACC-3') and ACTTRR1 (5'-CGTCTAGAACGAATCG-3'), which is the same probe used previously for the identification of the cosmid clone pcCL4-1 (AB176941) (Miyamoto et al. 2008). After colony hybridization, BAC DNA was extracted from the positive clones as described previously (Sambrook and Russell 2001; Hatta et al. 2006). The insert of BAC clone $2 \mathrm{H} 2$ was sequenced to $5 \times$ redundancy by shotgun sequencing using a capillary DNA sequencer. Transcripts of ACTTS3 were amplified with or without addition of reverse transcriptase (Takara, Shiga, Japan by RT-PCR using the primer sets PKS1F3 (5'-GCTGA CGAGACTGTTG- $3^{\prime}$ ) and PKS1R3 (5'-CTGTGTTTCCCCAC AG-3') for transcript amplification of ACTTS3, and actinFor (5'-AGATGACACAGATTGTTTTCGAGACC-3') and actinRev (5'-AGTTGAAAGTGGTGACGTGGATACC-3') for actin gene (act1 of A. alternata) (AY748985). RT-PCR products were transferred to Hybond-N+ membrane using standard methods (Sambrook and Russell 2001) and detected with a DIG-PCRlabeled ACTTS3 probe prepared using the same primer sets used for RT-PCR with genomic DNA of SH20 as the template by the methods described previously (Isshiki et al. 2001; Masunaka et al. 2000, 2005; Katoh et al. 2006, 2007; Miyamoto et al. 2008, 2009). The region containing the entire ORF of ACTTS3 was amplified from DNA and cDNA of SH20 by PCR consisting of 30 cycles (denaturing for $1 \mathrm{~min}$ at $95^{\circ} \mathrm{C}$, primer annealing for $1 \mathrm{~min}$, and extension for 1 min per $1 \mathrm{~kb}$ of amplicon at $\left.72^{\circ} \mathrm{C}\right)$ using multiple primer sets of PKS1F1 ( $5^{\prime}-\mathrm{T}$ GGATCCTGCTCAACG-3') and PKS1R1 (5'-ATTGGCGGG TATGGTG-3'), 2H2C27R1 (5'-GATGTGCAGGCCTAGCA-3') and 2H2C27F1 (5'-AGATGCAGTCCGACCG-3'), PKS1F2 (5'TGCGCCGAACCTTATC-3') and PKS1R2 (5'-TGGCGGCA ACTTCACA-3'), PKS1F3 (5'-GCTGACGAGACTGTTG-3') and C27Fe2 (5'-CAACGTGCGTGTA-3'), C27Rm2 (5'-TTCG AGCTCAACATCCAGGT-3') and C26Rm (5'-GCTTTGTATC GTATGCC-3'), 2H2C26F1 (5'-TTAGTCAGGCGGTCGA-3') and 2H2C26R1 (5'-TGCAGCAAGTCGGACT-3'), and PKS1F4 (5'-GACACCGCGAATCATG-3') and PKS1R4 (5'-CTGGCA ACGTACCCAA-3'). The transcription initiation site was identified with PKS1R6 (5'-CGCGCATCTGCAGATCCCACC AATCGGA-3') and GeneRacerTM 5' Primer, and PKS1R5 (5'CAACAAACACAGACGTGTGGGTACCCTGC-3') and GeneRacerTM 5' Nested Primer for nested PCR by 5' RACE with the GeneRacer kit (Invitrogen) according to the manufacturer's instructions. The transcription termination site was identified with PKS1F5 (5'-GGTGGTCAATCCAATTACCACG-3') and GeneRacerTM 3' Primer, and PKS1F6 (5'-CGTGCTCGAGGT CTTGCTGCATCGGTCA-3') and GeneRacerTM 3' Nested Primer for nested PCR by $3^{\prime}$ RACE. Amplicons were subcloned into pT7Blue-2 vector (Novagen, Madison, WI, U.S.A.) following the manufacturer's instructions. Sequencing was performed by the dideoxy chain termination method (Sanger et al. 1977) using an ABI PRISM dye termination cycle sequencing ready reaction kit (Applied Biosystems, Foster City, CA, U.S.A.) and an automated fluorescent DNA sequencer (Model 310; Applied Biosystems). Sequence identities were estimated using FASTA searches at the DNA Databank of Japan, and domain organizations were estimated using Pfam program (Finn et al. 2008).

\section{PFGE and hybridization.}

Preparation of chromosomes from fungal protoplasts and PFGE was carried out according to methods described previously (Hatta et al. 2002; Masunaka et al. 2005; Miyamoto et al. 2008, 2009). Running conditions (duration, voltage, and linear gradient of switching time) in a PFGE apparatus (CHEF-DRII; Bio-Rad, Richmond, CA, U.S.A.) consisted of $0.5 \times$ Tris-borate-EDTA (Sambrook and Russell 2001) at $8^{\circ} \mathrm{C}$ in $0.8 \%$ agarose gel (Seakem Gold agarose; BioWhittaker Molecular Applications) for $25 \mathrm{~h}$ at $1.6 \mathrm{~V} / \mathrm{cm} / 500 \mathrm{~s}, 30 \mathrm{~h}$ at 1.6 $\mathrm{V} / \mathrm{cm} / 1,000 \mathrm{~s}, 50 \mathrm{~h}$ at $1.6 \mathrm{~V} / \mathrm{cm} / 1,500 \mathrm{~s}$, and $32 \mathrm{~h}$ at 1.6 $\mathrm{V} / \mathrm{cm} / 2,000 \mathrm{~s}$. Chromosomal DNA bands were visualized by ethidium bromide staining, and the gel was serially treated by soaking in $0.2 \mathrm{~N} \mathrm{HCl}$ for $20 \mathrm{~min}$ (to nick chromosomal DNA) and $0.4 \mathrm{~N} \mathrm{NaOH}$ plus $1.5 \mathrm{M} \mathrm{NaCl}$ for $1 \mathrm{~h}$ to transfer Hybond$\mathrm{N}+$ membrane (Amersham Biosciences, Piscataway, NJ, U.S.A.) by alkaline capillary transfer (Sambrook and Russell 2001 ) with $0.4 \mathrm{~N} \mathrm{NaOH}$ and $1.5 \mathrm{M} \mathrm{NaCl}$ for $48 \mathrm{~h}$. The membrane was baked at $80^{\circ} \mathrm{C}$ for $2 \mathrm{~h}$ to fix the DNA and hybridized at $68^{\circ} \mathrm{C}$ overnight with ACTTS3 probes. Hybridization and washes were conducted under high-stringency conditions according to the manufacturer's instructions as described previously (Masunaka et al. 2005).

\section{Plasmid vector constructs and fungal transformation.}

The gene replacement vector ACTTS3hph was constructed using two partial regions of ACTTS 3 sequences amplified by PCR with the primer set of PKS1F10HindIII (5'-CCCAAG CTTGGGTGTGAAGTTGCCGCCA-3') and PKS1R3EcoRV (5'-GATATCCTGTGTTTCCCCACAG-3'), and PKS1F11 BamHI (5'-CGGGATCCCGTGTGGTTGACCAAGGG-3') and PKS1R10KpnI (5'-GGGGTACCCCGACATTTGCGGTC GCA-3'), containing HindIII, EcoRV, BamHI, and KpnI sites at the ends of their respective PCR-amplified sequences, using genomic DNA of $\mathrm{SH} 20$ as the template. Partial ACTTS3 sequences amplified by PCR were subcloned into the subcloning vector pT7Blue-2 (Novagen). An $h p h$ cassette (Cullen et al. 1987) from pSH75 (Kimura and Tsuge 1993) was inserted into pT7Blue-2 vector at EcoRV and BamHI sites and the constructed vector was named pAHY. The partial ACTTS3 sequence (PCR product using PKS1F11BamHI and PKS1R10KpnI) in pT7Blue-2 vector was subcloned into the pAHY at BamHI and $K p n I$. The pT7Blue-2 vectors containing the $h p h$ cassette and the partial ACTTS3 sequence were digested with HindIII and $E c o \mathrm{RV}$, and were ligated with another partial ACTTS3 sequence (PCR product using PKS1F10HindIII and PKS1R3EcoRV) digested with the same restriction enzymes to produce the gene replacement vector named ACTTS3hph. The vector was digested with NotI and KpnI and linearized before transformation to isolate $\mathrm{SH} 20$. 
For the second disruption of ACTTS3, ACTTS3NPTII was constructed using a partial sequence of ACTTS3 amplified by PCR with the primer set PKS1F12NotI (5'-GCGGCCGCCCA GACTGTCTGGGA-3') and PKS1R11BamHI (5'-CGGGATC CCGCAAGTAGCCACCAGGT-3') containing NotI and BamHI sites at the end of the PCR product using genomic DNA of SH20 as the template. Partial ACTTS3 sequences amplified by PCR were first subcloned into the subcloning vector pT7Blue-2 (Novagen). The geneticin resistance gene NPTII was amplified with the primer set of NPTIIF1EcoRI (5'-GGAATTCCAT GATTGAACAAGATGGATTGC-3') and NPTIIR1EcoRI (5'GGAATTCCTCAGAAGAACTCGTCAAGAA-3') containing EcoRI sites at the ends using pII99 vector (Namiki et al. 2001) as the template, and the product was subcloned in the Alternaria overexpression vector pAOEIK (Katoh et al. 2007) at the EcoRI site of pAOEIK, at the region between the promoter and terminator sequences of Aspergillus nidulans trpC, and the vector was named pAGY. The partial ACTTS3 sequence subcloned in the pT7Blue-2 vector was then digested with Not I and $B a m \mathrm{HI}$, and subcloned into the pAGY at the same restriction sites to complete the construction of ACTTS3NPTII vector. These vector constructs of ACTTS3hph and ACTTS3NPTII were confirmed by sequencing.

Protoplast preparation and transformation of isolate $\mathrm{SH} 20$ were carried out using methods described previously (Isshiki et al. 2001, 2003; Katoh et al. 2006, 2007; Miyamoto et al. 2008, 2009). Protoplasts $\left(1 \times 10^{7}\right)$ were transformed with $3 \mu \mathrm{g}$ of each vector. Colonies were transferred to PDA plates containing hygromycin B $(50 \mu \mathrm{g} / \mathrm{ml})$ or geneticin $(200 \mu \mathrm{g} / \mathrm{ml})$ and grown at $24^{\circ} \mathrm{C}$ for 5 to 7 days, and single-spore cultures were recovered as described previously (Isshiki et al. 2001, 2003; Katoh et al. 2006, 2007; Miyamoto et al. 2008, 2009).

\section{Detection of plasmid integration, toxin production, and pathogenicity.}

Integration patterns of transformation vectors in the genome of isolate SH20 were determined using genomic Southern blots and a DIG-labeled ACTTS3 probe. Hybridization and detection were conducted as described previously (Masunaka et al. 2000, 2005; Isshiki et al. 2001, 2003; Katoh et al. 2006, 2007; Miyamoto et al. 2008, 2009). Production of ACT-toxin in culture filtrates was determined by HPLC and bioassay by the following methods. Alternaria alternata isolate $\mathrm{SH} 20$ and its mutants were grown on PDA for examination of fungal growth and also in 200-ml flasks containing $50 \mathrm{ml}$ of AC-toxin medium (Kohmoto et al. 1979, 1993) for 25 to 30 days at $24^{\circ} \mathrm{C}$ for examination of ACT-toxin production in the culture filtrates. Culture filtrates were adsorbed to Amberlite XAD2, eluted with methanol, extracted twice with ethyl acetate, and suspended in methanol after passing through a Sep-Pak column plus C18 cartridge (Waters, Milford, MA, U.S.A.) as the protocol described previously (Masunaka et al. 2000, 2005; Miyamoto et al. 2008, 2009) for culture filtrate extract preparation for HPLC analysis. HPLC analysis of ACT-toxin was carried out on a Model PU-2080 HPLC system (Japan Spectroscopic, Tokyo) with a Develosil ODS-5 column (4.6 by 250 $\mathrm{mm}$; Noruma Chemical, Aichi, Japan) and a gradient solvent system composed of acetonitrile/water/acetic acid (linear gradient from 30:70:1 to $60: 40: 1$, vol/vol/vol) for $30 \mathrm{~min}$ at a flow rate of $1 \mathrm{ml} / \mathrm{min}$, with peak detection with an absorbance at $290 \mathrm{~nm}$ (Kohmoto et al. 1993; Masunaka et al. 2000, 2005; Miyamoto et al. 2008, 2009). Ethyl-acetate-extracted samples $(50 \mu \mathrm{l})$ from culture filtrates $(50 \mathrm{ml})$ were suspended in $1 \mathrm{ml}$ of methanol, passed through a Sep-Pak column Plus C18 Cartridge (Waters), and injected for HPLC analysis. Retention time and quantification of ACT-toxin was determined for each HPLC analysis using previously purified ACT-toxin I (Kohmoto et al. 1993; Masunaka et al. 2000, 2005; Miyamoto et al. 2008, 2009).

For pathogenicity assays, spores of each isolate were collected from mycelial mats grown in potato dextrose broth for 3 weeks and suspended in water at $1 \times 10^{5} \mathrm{spores} / \mathrm{ml}$ and spray inoculated on the lower surface of detached young leaves (midrib length: 3.0 to $4.0 \mathrm{~cm}$ ) of ACT-toxin-sensitive citrus cv. Iyokan as described previously (Masunaka et al. 2000, 2005; Miyamoto et al. 2008, 2009). Leaves were incubated in a moist chamber for $48 \mathrm{~h}$ at $24^{\circ} \mathrm{C}$ in the dark, and the development of leaf spots and necrosis was monitored.

\section{ACKNOWLEDGMENTS}

We thank L. W. Timmer for continuous support of this project and for providing Florida strains of A. alternata and M. Kodama and H. Otani, Tottori University, for valuable discussions and for providing strain O-94. This work was supported by grants for scientific research on priority area (S) (21228001) and in part (19108001) from the Japanese Society for Promotion of Sciences; from the Ministry of Education, Culture, Sports, Science, and Technology of Japan; Precursory Research for Embryonic Science and Technology from Japan Science and Technology Agency; and Special Coordination Funds for Promoting Sciences from Ministry of Education, Culture, Sports, Science, and Technology of Japan.

\section{LITERATURE CITED}

Abe, Y., Suzuki, T., Ono, C. Iwamoto, K., Hosobuchi, M., and Yoshikawa, H. 2002. Molecular cloning and characterization of an ML-236B (compactin) biosynthetic gene cluster in Penicillium citrinum. Mol. Genet. Genomics. 267:636-646.

Akamatsu, H., Itoh, Y., Kodama, M., Otani, H., and Kohmoto, K. 1997. AAL-Toxin-deficient mutants of Alternaria alternata tomato pathotype by restriction enzyme-mediated integration. Phytopathology 87:967972.

Akamatsu, H., Taga, M., Kodama, M., Johnson, R., Otani, H., and Kohmoto, K. 1999. Molecular karyotypes for Alternaria plant pathogens known to produce host-specific toxins. Curr. Genet. 35:647-656.

Akamatsu, H., Otani, H., and Kodama, M. 2003. Characterization of a gene cluster for host-specific AAL-toxin biosynthesis in the tomato pathotype of Alternaria alternata. Fungal Genet. Newsl. 50:355.

Akimitsu, K., Peever, T. L., and Timmer, L. W. 2003. Molecular, ecological and evolutionary approaches to understanding Alternaria diseases of citrus. Mol. Plant Pathol. 4:435-446.

Beck, J., Ripka, S., Siegner, A., Schiltz, E., and Schweizer, E. 1990. The multifunctional 6-methylsalicylic acid synthase gene of Penicillium patulum. Eur. J. Biochem. 192:487-498.

Covert, S. F. 1998. Supernumerary chromosomes in filamentous fungi. Curr. Genet. 33:311-319.

Cullen, D., Leong, S. A., Wilson, L. J., and Henner, D. J. 1987. Transformation of Aspergillus nidulans with the hygromycin-resistance gene, hph. Gene 57:21-26.

Feng, G., and Leonard, T. J. 1995. Characterization of the polyketide synthase gene ( $p k s L 1)$ required for aflatoxin biosynthesis on Aspergillus parasiticus. J. Bacteriol. 177:6246-6254.

Finn, R. D., Tate, J., Mistry, J., Coggill, P. C., Sammut, J. S., Hotz, H. R., Ceric, G.., Forslund, K., Eddy, S. R., Sonnhammer, E. L., and Bateman, A. 2008. The Pfam protein families database. Nucleic Acids Res. 36:D281-D288.

Harimoto, T., Hatta, R., Kodama, M., Yamamoto, M., Otani, H., and Tsuge, T. 2007. Expression profiles of genes encoded by the supernumerary chromosome controlling AM-toxin biosynthesis and pathogenicity in the apple pathotype of Alternaria alternata. Mol. PlantMicrobe Interact. 12:1463-1476.

Harimoto, T., Tanaka, T., Kodama, M., Yamamoto, M., Otani, H., and Tsuge, T. 2008. Multiple copies of AMT2 are prerequisite for the apple pathotype of Alternaria alternata to produce enough AM-toxin for expressing pathogenicity. J. Gen. Plant Pathol. 74:222-229.

Hatta, R., Ito, K., Hosaki, Y., Tanaka, T., Tanaka, A., Yamamoto, M., Akimitsu, K., and Tsuge, K. 2002. A conditionally dispensable chromosome controls host-specific pathogenicity in the fungal plant pathogen Alternaria alternata. Genetics 161:59-70.

Hatta, R., Shinjo, A., Ruswandi, S., Kitani, K., Yamamoto, M., Akimitsu, K., and Tsuge, T. 2006. DNA transposon fossils present on the conditionally dispensable chromosome controlling AF-toxin biosynthesis and pathogenicity of Alternaria alternata. J. Gen. Plant Pathol. 72:210-219. 
Hopwood, D. A. 1997. Genetic contributions to understanding polyketide synthases. Chem. Rev. 97:2465-2497.

Isshiki, A., Akimitsu, K., Yamamoto, M., and Yamamoto H. 2001. Endopolygalacturonase is essential for citrus black rot caused by Alternaria citri but not brown spot caused by Alternaria alternata. Mol. PlantMicrobe Interact. 14:749-757.

Isshiki, A., Ohtani, K., Kyo, M., Yamamoto, H., and Akimitsu, K. 2003. Green fluorescent detection of fungal colonization and endopolygalacturonase gene expression in the interaction of Alternaria citri with citrus. Phytopathology 93:768-773.

Ito, K., Tanaka, Hatta, R., Yamamoto, M., Akimitsu, K., and Tsuge, T. 2004. Dissection of the host range of the fungal plant pathogen Alternaria alternata by modification of secondary metabolism. Mol. Microbiol. 52:399-411.

Johnson, R. D., Johnson, L., Itoh, Y., Kodama, M., Otani, H., and Kohmoto, K. 2000. Cloning and characterization of a cyclic peptide synthetase gene from Alternaria alternata apple pathotype whose product is involved in AM-toxin synthesis and pathogenicity. Mol. Plant-Microbe Interact. 13:742-753.

Katoh, H., Isshiki, A., Masunaka, A., Yamamoto, H., and Akimitsu, K 2006. A virulence-reducing mutation in the postharvest citrus pathogen Alternaria citri. Phytopathology 96:934-940.

Katoh, H., Nalumpang, S., Yamamoto, H., and Akimitsu, K. 2007. Overexpression of citrus polygalacturonase-inhibiting protein in citrus black rot pathogen Alternaria citri. J. Plant Physiol. 164:527-535.

Kennedy, J., Auclair, K., Kendrew, S. G., Park, C., Vederas, J. C., and Hutchinson, C. R. 1999. Modulation of polyketide synthase activity by accessory proteins during lovastatin biosynthesis. Science 284:13681372.

Kimura, N., and Tsuge, T. 1993. Gene cluster involved in melanin biosynthesis of the filamentous fungus Alternaria alternata. J. Bacteriol. 175:4427-4435.

Kohmoto, K., and Otani, H. 1991. Host recognition by toxigenic plant pathogens. Experientia 47:755-764.

Kohmoto, K., Scheffer, R. P., and Whiteside, J. O. 1979. Host-selective toxins from Alternaria citri. Phytopathology 69:667-671.

Kohmoto, K., Akimitsu, K., and Otani, H. 1991. Correlation of resistance and susceptibility of citrus to Alternaria alternata with sensitivity to host-specific toxins. Phytopathology 81:719-722.

Kohmoto, K., Itoh, Y., Shimomura, N., Kondoh, Y., Otani, H., Kodama, M., Nishimura, S., and Nakatsuka, S. 1993. Isolation and biological activities of two host-specific toxins from the tangerine pathotype of Alternaria alternata. Phytopathology 83:495-502.

Kroken, S., Glass, N. L., Taylor, J. W., Yoder, O. C., and Turgeon, B. G. 2003. Phylogenomic analysis of type I polyketide synthase genes in pathogenic and saprobic ascomycetes. Proc. Natl. Acad. Sci. U.S.A. 100:15670-15675.

Kusaba, M., and Tsuge, T. 1994. Nuclear ribosomal DNA variation and pathogenic specialization in Alternaria fungi known to produce hostspecific toxins. Appl. Environ. Microbiol. 60:3055-3062.

Kusaba, M., and Tsuge, T. 1995. Phologeny of Alternaria fungi known to produce host-specific toxins on the basis of variation in internal transcribed spacers of ribosomal DNA. Curr. Genet. 28:491-498.

Linden, H., and Macino, G. 1997. White collar 2, a partner in blue-light signal transduction, controlling expression of light-regulated genes in Neurospora crassa. EMBO (Eur. Mol. Biol. Organ.) J. 16:98-109.

Marzluf, G. A. 1993. Genetic regulation of nitrogen metabolism in the fungi. Microbiol. Mol. Biol. Rev. 61:1-17.

Masunaka, A., Tanaka, A., Tsuge, T., Peever, T. L., Timmer, L. W. Yamamoto, M., Yamamoto, H., and Akimitsu, K. 2000. Distribution and characterization of $A K T$ homologs in the tangerine pathotype of Alternaria alternata. Phytopathology 90:762-768.

Masunaka, A., Ohtani, K., Peever, T. L., Timmer, L. W., Tsuge, T., Yamamoto, M., Yamamoto, H., and Akimitsu, K. 2005. An isolate that is pathogenic to both tangerines and rough lemon and produces two hostselective toxins, ACT- and ACR-toxins. Phytopathology 95:241-247.

Miyamoto, Y., Masunaka, A., Tsuge, T., Yamamoto, M., Ohtani, K., Fukumoto, T., Gomi, K., Peever, T. L., and Akimitsu, K. 2008. Functional analysis of a multicopy host-selective ACT-toxin biosynthesis gene in the tangerine pathotype of Alternaria alternata using RNA silencing. Mol. Plant-Microbe Interact. 21:1591-1599.

Miyamoto, Y., Ishii, Y., Honda, A., Masunaka, A., Tsuge, T., Yamamoto, M., Ohtani, K., Fukumoto, T., Gomi, K., Peever, T. L., and Akimitsu,
K. 2009. Function of genes encoding acyl-CoA synthetase and enoylCoA hydratase for host-selective ACT-toxin biosynthesis in the tangerine pathotype of Alternaria alternata. Phytopathology 99:369-377.

Moss, S. J., Martin, C. J., and Wilkinson, B. 2004. Loss of co-linearity by modular polyketide synthases: a mechanism for the evolution of chemical diversity. Nat. Prod. Rep. 21:575-593.

Nakashima, T., Ueno, T., Fukami, H., Taga, T., Masuda, H., Osaki, K., Otani, H., Kohmoto, K., and Nishimura, S. 1985. Isolation and structure of AK-toxin I and II, host-specific phytotoxic metabolites produced by Alternaria alternata Japanese pear pathotype. Agric. Biol. Chem. 49:807-815.

Nakatsuka, S., Ueda, K., Goto, T., Yamamoto, M., Nishimura, S., and Kohmoto, K. 1986. Structure of AF-toxin II, one of the host-specific toxins produced by Alternaria alternata strawberry pathotype. Tetrahedron Lett. 27:2753-2756.

Namiki, F., Matsunaga, M., Okuda, M., Inoue, I., Nishi, K., Fujita, Y., and Tsuge, T. 2001. Mutation of an arginine biosynthesis gene causes reduced pathogenicity in Fusarium oxysporum f. sp. melonis. Mol. PlantMicrobe Interact. 14:580-584.

Nishimura, S., and Kohmoto, K. 1983. Host-specific toxins and chemical structures from Alternaria species. Annu. Rev. Phytopathol. 21:87-116.

Peever, T. L., Canihos, Y., Olsen, L., Ibanez, A., Liu, Y. C., and Timmer, L. W. 1999. Population genetic structure and host specificity of Alternaria spp. causing brown spot of Minneola tangelo and rough lemon in Florida. Phytopathology 89:851-860.

Peever, T. L., Su, G., Carpenter-Boggs, L., and Timmer, L. W. 2004. Molecular systematics of citrus-associated Alternaria species. Mycologia 96:119-134.

Rangan, V. S., Oskouian, B., and Smith, S. 1996. Identification of an inverted CCAAT box motif in the fatty-acid synthase gene as an essential element for mediation of transcriptional regulation by cAMP. J. Biol. Chem. 271:2307-2312.

Ruswandi, S., Kitani, K., Akimitsu, K., Tsuge, T., Shiraishi, T., and Yamamoto, M. 2005. Structural analysis of cosmid clone pcAFT-2 carrying AFT10-1 encoding an acyl-CoA dehydrogenase involved in AF-toxin production in the strawberry pathotype of Alternaria alternata. J. Gen. Plant Pathol. 71:107-116.

Sambrook J., and Russell D. W. 2001. Molecular Cloning: A Laboratory Manual, 3rd ed. Cold Spring Harbor Laboratory, Cold Spring Harbor, NY, .U.SA.

Sanger, F., Nicklen, S., and Couldon, A. R. 1977. DNA sequencing with chain-terminating inhibitors. Proc. Natl. Acad. Sci. U.S.A. 74:54635467.

Scheffer, R. P., and Livingston, R. S. 1984. Host-selective toxins and their role in plant diseases. Science 223:17-21.

Tanaka, A., and Tsuge, T. 2000. Structural and functional complexity of the genomic region controlling AK-toxin biosynthesis and pathogenicity in the Japanese pear pathotype of Alternaria alternata. Mol. PlantMicrobe Interact. 13:975-986.

Tanaka, A., Shiotani, H., Yamamoto, M., and Tsuge, T. 1999. Insertional mutagenesis and cloning of the genes required for biosynthesis of the host-specific AK-toxin in the Japanese pear pathotype of Alternaria alternata. Mol. Plant-Microbe Interact. 12:691-702.

VanEtten, H. D., Jorgensen, S., Enkerli, J., and Covert, S. F. 1998. Inducing the loss of conditionally dispensable chromosomes in Nectria haematococca during vegetative growth. Curr. Genet. 33:299-303.

Walton, J. D. 1996. Host-selective toxins: agents of compatibility. Plant Cell 8:1723-1733.

Wolpert, T. J., Dunkle, L. D., and Ciuffetti, L. M. 2003. Host-selective toxins and avirulence determinants: what's in a name? Annu. Rev. Phytopathol. 40:251-285

Yang, G., Rose, M. S., Turgeon, B. G., and Yoder, O. C. 1996. A polyketide synthase is required for fungal virulence and production of the polyketide T-toxin. Plant Cell. 8:2139-2150.

Yoder, O. C. 1980. Toxins in pathogenesis. Annu. Rev. Phytopathol. 18:103-129.

\section{AUTHOR-RECOMMENDED INTERNET RESOURCES}

DNA Data Bank of Japan (DDBJ): www.ddbj.nig.ac.jp

Wellcome Trust Sanger Institute Pfam program:

www.sanger.ac.uk/Software/Pfam 